\title{
Monitoring same/different discrimination behavior in time and space: Finding differences and anticipatory discrimination behavior
}

\author{
DANIEL I. BRoOKS AND EDWARD A. WASSERMAN \\ University of Iowa, Iowa City, Iowa
}

\begin{abstract}
Discrimination behavior in a standard, two-alternative forced choice same/different task is usually measured by the pigeon's pecking one or the other of two arbitrary report areas. We found that pigeons make anticipatory, discriminative responses to the visual display during the stimulus observing period prior to the availability of the report areas; the spatial distribution of these anticipatory discriminative responses strongly correlated with the upcoming choice response. These anticipatory pecks provide evidence that the process of discrimination occurs well before the moment of choice and that key aspects of this process can be revealed by looking at the distribution of observing responses. We also manipulated the variability of the displayed items to study the nature of these anticipatory responses; again, the spatial distribution of responding during the stimulus observing period strongly correlated with the upcoming choice response. The distribution of these prechoice pecks supports the theory that pigeons search for differences in the displayed items. If differences are found, then pigeons prepare to report "different"; if not, then they report "same."
\end{abstract}

The ability to categorize a set of items as "same" or "different" is often deemed to be foundational to human cognition (Katz, Wright, \& Bodily, 2007; Wasserman \& Young, 2010). These abstract relations have been intensively studied because they are vital to adaptation in a complex and changing world; sensitivity to same and different relations allows us to draw important comparisons among the many objects and environments that we encounter.

Despite the key roles that sameness and differentness play in cognition and adaptive action (Wasserman, Young, \& Cook, 2004), the capacity to detect abstract relations was once believed to be a purely human competence, "which the Faculties of Brutes do by no means attain to" (Locke, 1690/1975, pp. 159-160). Nevertheless, research with nonhuman animals has shown that other primates (Katz, Wright, \& Bachevalier, 2002; Wasserman, Fagot, \& Young, 2001) and even pigeons (Wasserman, Hugart, \& Kirkpatrick-Steger, 1995; Wright \& Katz, 2006) exhibit sensitivity to abstract relations.

Although same and different are often assumed to be equally important conceptual "twins" (Delius, 1994), recent research has revealed that these two concepts may not be equally salient. Mounting evidence suggests that same and different relations are not equivalently discriminated.

The first piece of evidence concerns asymmetrical rates of learning in same/different tasks in which animals must peck at a particular set of items rather than at an arbitrary report area. For example, Blaisdell and Cook (2005) found that different ${ }^{+}$pigeons taught to peck at a pair of different items, but not to peck at a pair of same items, learned faster than same ${ }^{+}$pigeons trained to peck at a pair of same items, but not to peck at a pair of different items. Similarly, Castro, Kennedy, and Wasserman (2010) taught a single group of pigeons to peck at a same array or a different array of items depending on a superordinate color cue; the birds learned to peck "different" more quickly than they learned to peck "same." Finally, faster learning of different than of same relations was reported by Wasserman, Frank, and Young (2002), who gave pigeons a go/ no-go task: Different ${ }^{+}$pigeons taught "peck at different/ no peck at same" learned faster than did same ${ }^{+}$pigeons taught "peck at same/no peck at different." This set of experiments documents that, with some training procedures, same and different trials are not learned at equivalent rates.

The second piece of evidence is the asymmetrical behavioral effect of reducing the number of items. When pigeons are first trained with arrays containing 16 items and they are later tested with displays containing fewer items, discrimination falls (Brooks \& Wasserman, 2010; Young, Wasserman, \& Garner, 1997). Rather than symmetrically falling on same and different trials, pigeons continue to respond correctly on same trials, but they increasingly report "same" when shown an array of different items as the number of items in the display is reduced. On trials in which only 2 items are shown - same or differentpigeons persistently report "same." This peculiar pattern of behavior suggests that pigeons translate the categorical same/different discrimination into a continuous variability discrimination. If entropy, an information-theoretic

D. I. Brooks, daniel-brooks@uiowa.edu 
measure of variability, is used to characterize the training stimuli, then one finds that the entropy of a 16-item different display is as high as possible (4), whereas the entropy of a 16 -item same display is as low as possible (0). Reducing the number of items on same trials cannot decrease entropy, but reducing the number of items on different trials decreases entropy toward 0 .

A more recent model (Young, Ellefson, \& Wasserman, 2003), finding differences, offers an alternative approach to calculating variability, but it arrives at the same conclusion: As the number of items in the display falls, variability too falls. Critically, this model goes beyond entropy by envisioning the same/different task in terms of difference detection. The model characterizes the drop in discrimination as the number of items falls by hypothesizing that pigeons detect fewer differences; the fewer the differences, the greater the likelihood of their reporting "same."

\section{Categorization: Beyond Choice Behavior}

Recent researchers in cognitive science have analyzed discrimination behavior before a final choice response has been executed. For example, Spivey, Grosjean, and Knoblich (2005) used "mouse tracking" to measure changes in the behavior of people given a computer-based task before subjects actually made their selection in a two-alternative forced choice paradigm. The researchers discerned disparities in the trajectory of the mouse movements toward the correct alternative when people were given an easy or a difficult competitor, thereby revealing an online decision-making process that could be measured before the actual moment of choice. Relatedly, capturing prechoice eye movements in both human adults (Tanenhaus, Spivey-Knowlton, Eberhard, \& Sedivy, 1995) and infants (McMurray \& Aslin, 2005) has yielded important insights into language comprehension, categorization, and visual perception.

This kind of analysis has not been limited to human performance; data from pigeon go/no-go tasks have been similarly scrutinized. For example, Cook and Brooks (2009) deployed a technique in which separate portions of each multi-item trial were analyzed to reveal different temporal patterns of discrimination. The spatial location of pigeons' pecks has also been used to study other kinds of discrimination behavior. For example, pigeons selectively peck at the diagnostic feature in a feature-positive discrimination (Jenkins \& Sainsbury, 1970; Wasserman \& Anderson, 1974) or at category-specific information in a categorization task (Allan, 1993; Dittrich, Rose, Buschmann, Bourdonnais, \& Güntürkün, 2010).

To our knowledge, only one study (Cook, Levison, Gillett, \& Blaisdell, 2005) has examined the spatial distribution of initial pecks toward the location of an upcoming response in a forced choice task. There, the location of pigeons' first peck at a pictorial stimulus predicted choice accuracy in a study of long-term visual memory, thus providing the researchers with a diagnostic measure for the time course of picture recognition.

Therefore, in both humans and animals, detailed behavioral analyses have revealed that the discrimination process can be recorded before the actual behavior of making a final choice. Such prechoice measurement may be vital because it allows the ongoing recording of the discrimination process, possibly disclosing key insights into its nature. Carefully tracking spatial shifts in prechoice responding holds particular promise in both animal and human research, because such shifts strongly suggest prospective processing of an impending choice response. Because many animal studies require one or more observing responses before a final choice response is made, documenting the utility of this technique may have important implications for methods in comparative cognition and, more generally, behavioral studies in the neurosciences.

\section{Peck Location and \\ Same/Different Discrimination}

This methodological technique of tracking the location of the pigeon's observing response has not yet been applied to the study of multi-item same/different arrays. This technique is of particular interest to us because of a specific account of discrimination behavior - namely, that pigeons use a "finding differences" strategy to discriminate same from different arrays. This account hypothesizes that pigeons attend to local differences that are created when items are different from their surrounding neighbors and that sensitivity to these differences is paramount in the pigeon's ability to discriminate these displays.

The pigeons that we study in the present experiment were previously trained on a trial-unique same/different task (Brooks \& Wasserman, 2008). Here, birds are shown an array of identical or nonidentical trial-unique objects, and they are required to peck one key to indicate "same" and a second key to indicate "different." Because the stimuli are trial unique, all trials are conducted without the participation of exemplar memory; all training trials are transfer trials.

On each trial, we required the pigeons to complete a fixed number of pecks to the display screen before offering them the choice responses. If pigeons utilize a difference detection strategy and they detect a strong difference signal in a multi-item display, then we might see them distribute their display-directed responses toward the side of the display where the upcoming "different" choice response will be made - even before the "same" and "different" choice areas are presented. If pigeons do not detect a strong difference signal, then we might not see them skew their display-directed responses toward either side of the display. The key behavioral measure in this analysis is the spatial distribution of pecking during the stimulus observation period.

The "finding differences" account also predicts how behavior should vary as a function of intermediate levels of display variability. Beyond varying the number of displayed items, one can also manipulate display variability by changing the mixture of same and different items in an array (Figure 1, bottom row). When the variability of the multi-item array is manipulated, choice behavior also changes; pigeons classify more-variable displays as 
Different

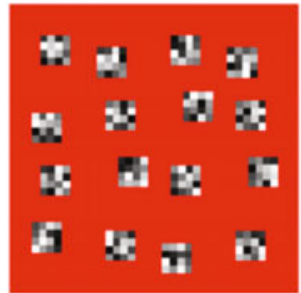

Same

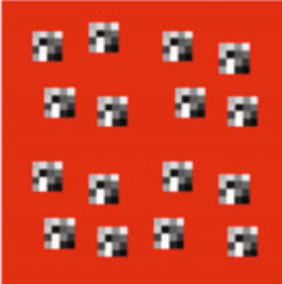

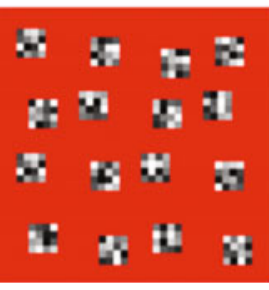

$14 \mathrm{D} / 2 \mathrm{~S}$

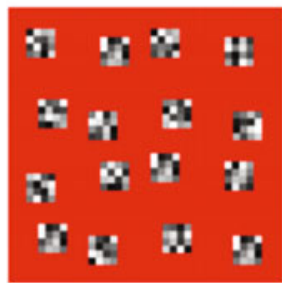

$12 \mathrm{D} / 4 \mathrm{~S}$

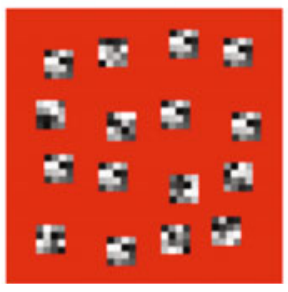

$8 \mathrm{D} / 8 \mathrm{~S}$

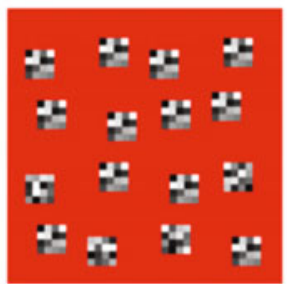

$4 \mathrm{D} / 12 \mathrm{~S}$

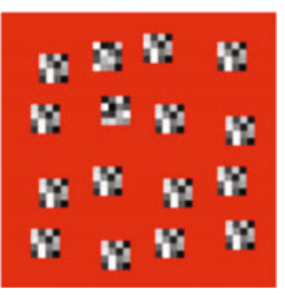

$2 \mathrm{D} / 14 \mathrm{~S}$

Figure 1. Sample stimuli from the trial-unique same/different task. Top row: Baseline same/different trials. Bottom row: Mixture trials, which increase in variability from left to right. " $S$ " denotes the number of identical and " $D$ " denotes the number of nonidentical stimuli in each mixture display. Available online in color at http://pbr.psychonomic-journals .org/content/current.

"different" and less-variable displays as "same" (Young \& Wasserman, 1997). To see whether prechoice pecking behavior is also sensitive to display variability, we varied the mixture of same and different items in the display.

\section{METHOD}

\section{Subjects}

Four feral pigeons (Columba livia) were kept at $85 \%$ of their free-feeding weights. The birds had earlier learned the trial-unique same/different discrimination task devised by Brooks and Wasser$\operatorname{man}(2008)$.

\section{Apparatus}

The pigeons were trained in four operant conditioning chambers (Gibson, Wasserman, Frei, \& Miller, 2004). The experimental stimuli were presented on an LCD monitor behind a resistive touchscreen. A food dispenser delivered $45-\mathrm{mg}$ pellets into a small cup on the rear wall. A houselight provided ambient illumination during sessions.

\section{Stimuli}

Baseline training. Each trial stimulus was a $9.5 \times 9.5 \mathrm{~cm}$ display in the center of the screen (Figure 1, top row), which subtended between 87 and 115 deg of visual angle. Each display contained $16(1 \times 1 \mathrm{~cm})$ mosaics roughly organized in a $4 \times 4$ fashion, with each mosaic being a neat $4 \times 4$ matrix and with each cell $(0.25 \times$ $0.25 \mathrm{~cm}$ ) randomly filled with 1 of 16 luminance levels. These 16 luminances were equally spaced along a monochromatic spectrum. To ensure that the displays would be of equal overall luminance (socalled isoluminant displays), each of the 16 luminances was used once in each mosaic.

During baseline training, two kinds of displays were shown: same displays, in which each mosaic was constructed from an identical luminance pattern, and different displays, in which each mosaic was constructed from nonidentical luminance patterns (Figure 1, top row). During baseline training trials, 16 mosaics were placed on a red square background and arranged so that the stimuli in each row and column were randomly staggered, eliminating any simple perceptual strategy for solving the task (Wasserman, Frank, \& Young, 2002; Young \& Wasserman, 2001).
Mixture testing. During mixture testing, five additional types of displays were used (Figure 1, bottom row; modeled after Young \& Wasserman, 1997); they were mixtures of some identical and some nonidentical items, each of which was both trial unique and isoluminant. The five mixtures were: $14 \mathrm{D} / 2 \mathrm{~S}$ (14 different/2 same), $12 \mathrm{D} / 4 \mathrm{~S}$, $8 \mathrm{D} / 8 \mathrm{~S}, 4 \mathrm{D} / 12 \mathrm{~S}$, and $2 \mathrm{D} / 14 \mathrm{~S}$. All of the choices to these testing stimuli were followed by nondifferential food reinforcement.

\section{Procedure}

Each trial started with the presentation of a white square $(7 \times$ $7 \mathrm{~cm}$ ) containing a central black cross. After one peck anywhere within the square, a single $9.5 \times 9.5 \mathrm{~cm}$ display was presented until the bird completed a fixed number of pecks (FR); pecks were counted only if they occurred within the display. The bird had to discriminate same from different displays by pecking one of two $2.5 \times 2.5 \mathrm{~cm}$ buttons (a circle and a triangle) to the left and right of the display (counterbalanced across birds) that onset immediately after the completion of the FR. During baseline trials, correct choices produced one to two food pellets; incorrect choices were punished by a dark timeout (ranging from 30 to $35 \mathrm{sec}$ ), followed by one or more unscored correction trials, which repeated until the bird made the correct response. After a correct choice, an 8-sec intertrial interval followed.

Daily sessions comprised 170 randomly ordered baseline and mixture testing trials. During each session, each type of baseline training trial was presented 75 times; each type of mixture trial was presented 4 times. A total of 50 sessions of data were collected for each bird.

\section{RESULTS}

\section{Baseline Same/Different Trials}

Figure 2B shows that accuracy on baseline training trials was high, with individual pigeons averaging $87.3 \%$ (88B), $94.9 \%(11 \mathrm{Y}), 75.8 \%(36 \mathrm{R})$, and $97.4 \%(81 \mathrm{~W})$ correct on same trials and $93.2 \%$ (88B), 92.5\% (11Y), 96.1\% (36R), and $92.8 \%(81 \mathrm{~W})$ correct on different trials. Two birds chose more accurately on same trials, whereas 2 birds chose more accurately on different trials. 


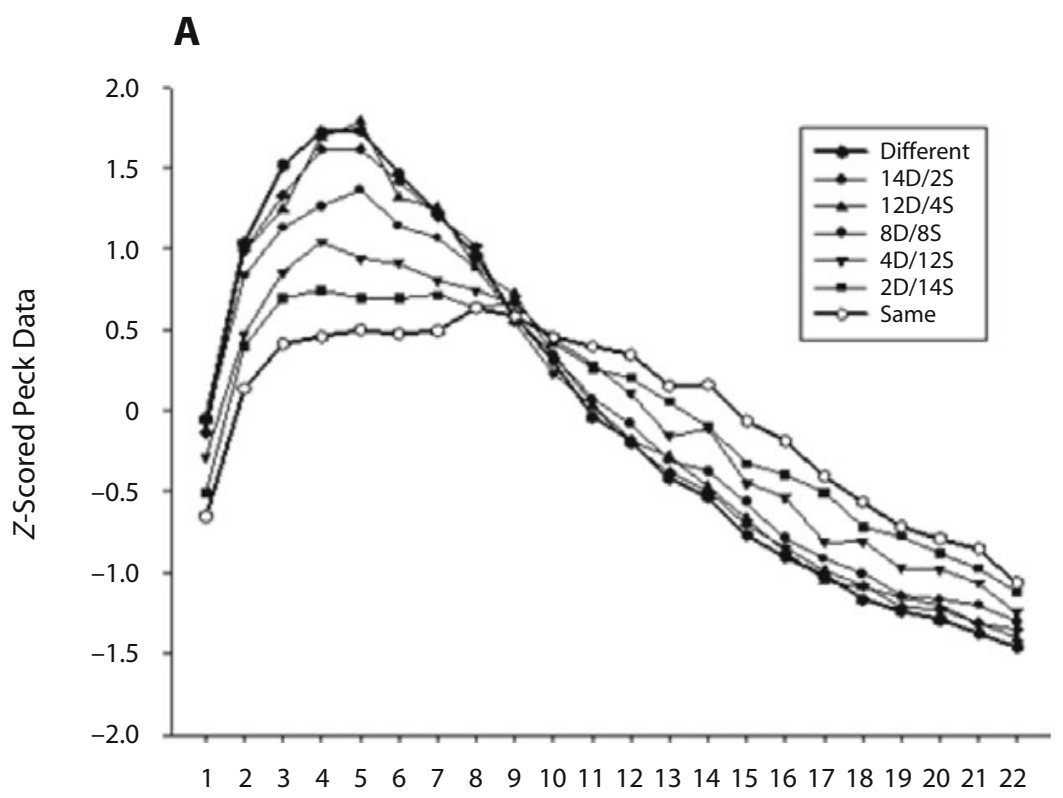

Position on Center Stimulus

B

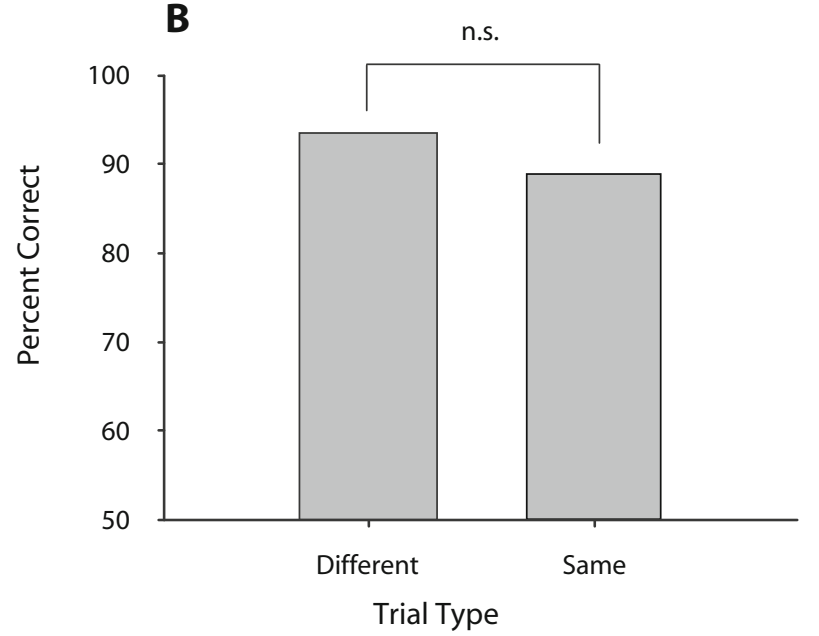

C

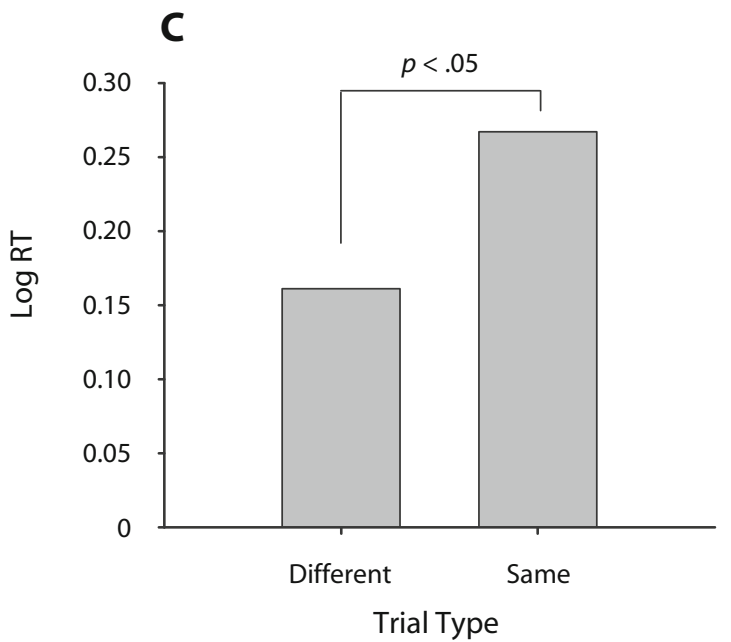

Figure 2. Panel A depicts data collected during the stimulus observation period. Panels $B$ and $\mathbf{C}$ depict data collected during the choice response period. Panel A shows $Z$-scored histograms of the spatial distributions of pecks during same, different, or mixture trials. This graph has been calibrated so that the "different" report key is located on the left and the "same" report key is located on the right, despite counterbalanced assignments among the 4 pigeons. Panel B shows choice accuracy on same and different trials. Panel $\mathrm{C}$ shows log-transformed choice latencies on correct same and different trials.

Figure 2A depicts the spatial distributions of all FR pecks on same and different trials during the stimulus observation period on correct trials. These distributions have been aligned so that the leftmost points on the distributions are closest to the upcoming "different" report response position and the rightmost points on the distributions are closest to the upcoming "same" report response position, regardless of the birds' actual response assignments (counterbalanced across pigeons; the distributions for 2 birds were thus mirror reversed). Each bird's peck distribution has been standardized by converting absolute numbers of pecks into $z$ scores determined by that bird's mean and standard deviation in pecking responses.

Pecks on different trials formed a highly peaked distribution that was strongly shifted toward the location of the upcoming "different" response, whereas pecks on same trials formed a flatter and more symmetrical distribution. Although the birds were generally more likely to peck near the "different" response location on all of the trials, they more tightly concentrated their pecking behavior toward the "different" response location when they were shown 
displays of nonidentical items than when they were shown displays of identical items.

To statistically analyze the evident disparity between the spatial distributions of pecks on same and different trials, we used the rho statistic - a nonparametric measure of the disparity between response distributions that has often been used to analyze disparities in response rates (e.g., Herrnstein, Loveland, \& Cable, 1976). Rho is formally equivalent to the area under the ROC curve in signal detection theory (Bamber, 1975); it is computed by first calculating the Mann-Whitney $U$ statistic for the two distributions and then dividing $U$ by its maximum possible value, $N_{1} * N_{2}$. The minimum value for rho, with entirely overlapping distributions, is .5; the maximum value for rho, with entirely nonoverlapping distributions, is 1.0.

The mean value of rho across all of the FR pecks on same and different trials was .62. A one-tailed $t$ test against chance responding $(.50)$ revealed that the pigeons generated significantly different peck distributions on same and different trials $[t(3)=3.10, p<.05]$. Rho was next computed for the distribution of pecks for each response in the FR sequence and entered into a repeated measures ANOVA (FR position $\times$ bird as a random factor). This analysis revealed a significant main effect of FR position $[F(14,42)=3.47$, $p<.05$ ], confirming the reliability of the rising values of rho from .57 to .59 to .60 to .61 to .62 for the first five pecks in the FR; thereafter, rho remained at or above 63 .

If, as Figure 2A suggests, the pigeons were generally more likely to concentrate their FR pecks in the direction of the "different" response location and they were especially prone to do so on different trials, then it might also be the case that "different" report responses would be executed faster than "same" report responses. This trend should hold because the pigeons ought to be located closer to the "different" report area on different trials than to the "same" report area on same trials. Consistent with this expectation, the pigeons were faster to respond "different" than to respond "same" on correct report trials (Figure 2C): Mean RT on different trials was $1.77 \mathrm{sec}$, whereas mean RT on same trials was $2.11 \mathrm{sec}$. Log RTs from correct trials were entered into an ANOVA (bird $\times$ trial type $\times$ five-session blocks as a random factor), which yielded a significant main effect of trial type $[F(1,9)=253.08, p<.001]$.

\section{Mixture Trials}

During mixture trials, shown in Figure 3B, pigeons increasingly chose "different" as the variability of the mixture increased. A two-parameter logarithmic function that modeled the mean choice data from the average of the 4 pigeons as a function of display entropy yielded $R^{2}=$ .994 , indicating a robust fit, consistent with prior mathematical descriptions of the mixture manipulation (Young \& Wasserman, 2001).

As with baseline trials, we also analyzed the FR responses that pigeons made in the observation period. The spatial distribution of these responses is plotted in Figure $2 \mathrm{~A}$. The concentration of pecking toward the "different" response covaried with display variability. For example, the small rise in variability from the same $(0 \mathrm{D} / 16 \mathrm{~S})$ display to the $2 \mathrm{D} / 14 \mathrm{~S}$ display prompted a modest increase

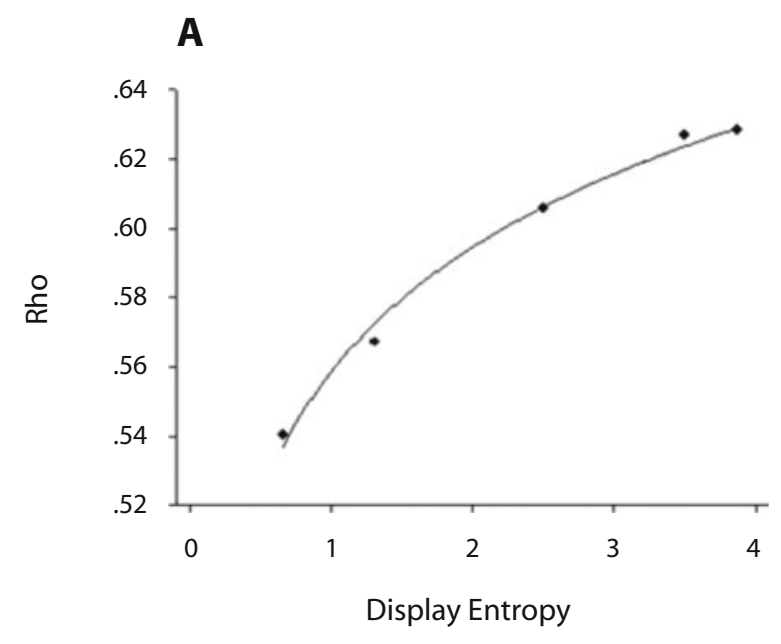

\section{B}
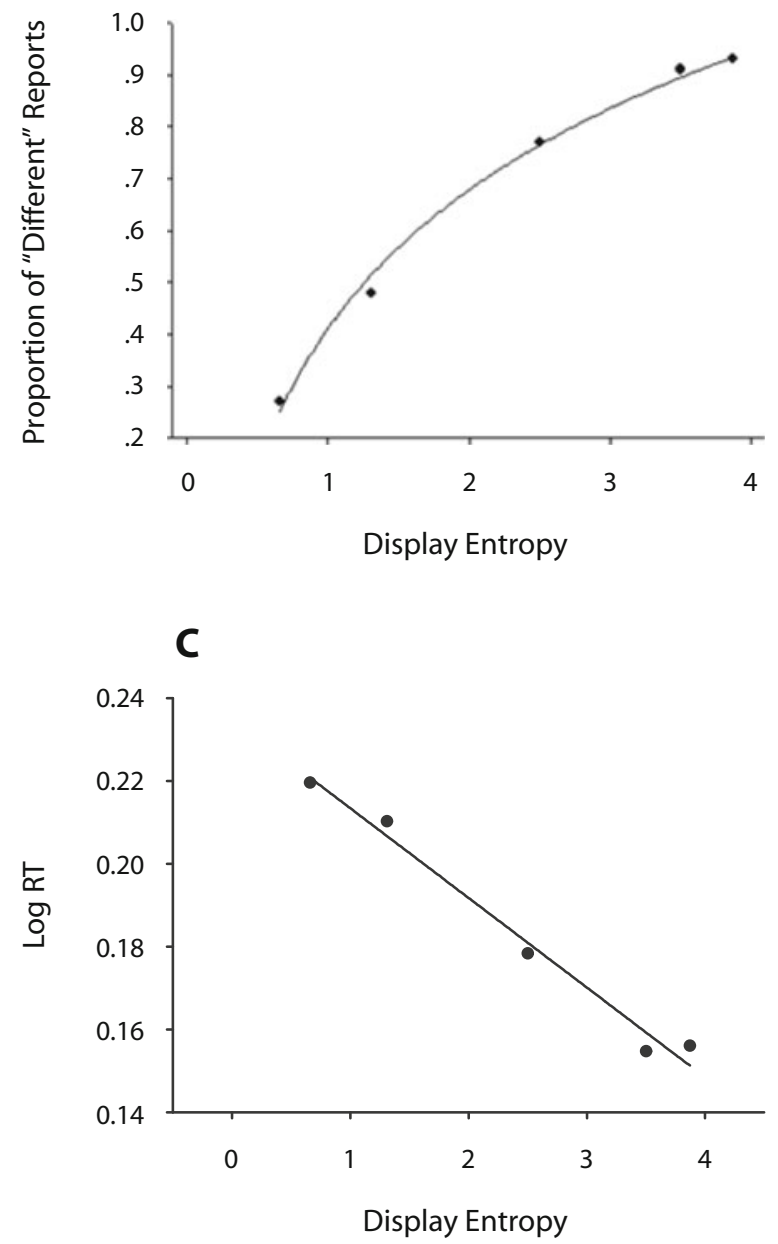

Figure 3. Summary data for the mixture trials. Panel A depicts data collected during the observation period; Panels $B$ and $C$ depict data collected during the choice response period. Panel A illustrates Herrnstein's rho statistic (see text for details) for assessing the disparity between each mixture distribution and the same baseline distribution. Panel B illustrates the proportion of "different" reports on mixture trials as a function of display entropy. Panel C illustrates log-transformed choice latency on mixture trials as a function of display entropy. 
in the concentration of pecking toward the "different" position; a further increase in variability to $4 \mathrm{D} / 12 \mathrm{~S}$ produced a further shift in the distribution. This trend continued for both $8 \mathrm{D} / 8 \mathrm{~S}$ and $12 \mathrm{D} / 4 \mathrm{~S}$ displays. The highly variable $12 \mathrm{D} / 4 \mathrm{~S}$ and $14 \mathrm{D} / 2 \mathrm{~S}$ display distributions were very similar to the distribution of pecks on different $(16 \mathrm{D} / 0 \mathrm{~S})$ trials, consistent with the increasing likelihood of "different" responses to these stimuli.

The spatial distribution of responses during the FR period was analyzed by calculating the disparity between each mixture distribution with the same baseline distribution using rho. These values are plotted in Figure 3A. The resulting rho statistic was then correlated with entropy; a logarithmic trend nicely fit the data with $R^{2}=.991$. A highly similar trend was observed when the disparity was calculated between each mixture distribution and the baseline different distribution; a logarithmic trend fit the data with $R^{2}=.996$. Therefore, the variability of the visual display was directly correlated with the distribution of pecks during the observation period - as variability rose, the mixture distribution became increasingly disparate from the distribution of pecks on same trials and increasingly similar to the distribution of pecks on different trials.

As with baseline same and different trials, we compared choice latencies on the various mixture trials. Consistent with the notion of difference detection and with the prior interpretation of RT disparities on baseline same and different trials, $\log$ RTs fell linearly $\left(R^{2}=.960\right)$ as the variability of the mixture display increased (Figure 3C).

\section{DISCUSSION}

Monitoring the spatial distribution of responses during the stimulus observation period may provide fresh insights into the nature of behavior in choice discrimination tasks. By measuring peck location during the FR, before the pigeons chose the "same" or "different" key, we discovered a high incidence of anticipatory responses, which predicted the location of the upcoming choice response. These anticipatory pecks were asymmetrically distributed; whereas the distribution of pecks on different trials revealed a large shift toward the "different" response, the distribution of pecks on same trials was much less skewed. These differential anticipatory distributions may divulge the psychological process that the birds use to discriminate these displays - namely, the pigeons may detect differences in the display. Our method of monitoring the birds' observing responses allowed us to measure this process before the birds ever made the required choice response.

Our success in analyzing the spatial distribution of responding may capitalize on several features of our same/ different task. First, all of the stimuli that will be presented on each trial are immediately available at the start of the FR, unlike in other tasks in which the animal must complete the FR to one picture and then choose between another, identical picture to report "same" and a "bailout" stimulus to report "different" (e.g., Wright \& Katz, 2006); in those tasks, pecks during the initial FR cannot be diagnostic because the same or different status of the second stimulus cannot be known during the FR. Second, our task involves choice keys that are always in fixed spatial locations, allowing us to observe spatial anticipation that would be impossible with a randomized-choice task (e.g., Wasserman, Young, \& Peissig, 2002). Third, it may be that our trial-unique stimuli are particularly well-suited to tracking the spatial distribution of pecking. Prior research has found that pigeons are likely to peck at the diagnostic features of familiar stimuli that indicate category membership (Allan, 1993), as well as to memorize repeating stimuli in same/different tasks despite the functional inadequacy of this strategy (Wasserman et al., 1995). Presenting novel stimuli on each trial may discourage the birds from directing pecks at particular stimuli in the display and instead encourage the birds to direct pecks toward the location of the upcoming choice response.

The systematic shift in the spatial distribution of pecking on mixture trials is further evidence that the birds directed their observing responses in accord with their upcoming choice responses. When display variability was manipulated, both rho and choice behavior varied according to a highly similar logarithmic function. Predictably, there was a nearly perfect linear correlation $\left(R^{2}=.990\right)$ between rho and choice for these distributions.

Both the spatial distribution of pigeons' observing responses and their final choice responses closely accorded with visual display entropy; these facts underscore the strong role that variability plays in pigeons' same/different discrimination behavior. However, although entropy effectively quantifies stimulus variability and dramatically accords with pigeons' behavior, it is not a psychological process. Any organic computation of entropy requires discriminating the frequency of the different kinds of objects in the entire display or at least large portions of it, certainly requiring item categorization and (at least shortterm) memory. "Finding differences," on the other hand, relies only on extracting local disparities, thereby eschewing complex areal calculations. This more localized processing is especially useful in accounting for sensitivity to variability with trial-unique stimuli, in which the (longterm) memorization of individual items is precluded.

\section{AUTHOR NOTE}

Comments concerning this article may be directed to D. I. Brooks, Psychology Department, E11 Seashore Hall, University of Iowa, Iowa City, IA 52242 (e-mail: daniel-brooks@uiowa.edu).

\section{REFERENCES}

Allan, R. W. (1993). Control of pecking response topography by stimulus-reinforcer and response-reinforcer contingencies. In H. P. Zeigler \& H.-J. Bischof (Eds.), Vision, brain, and behavior in birds (pp. 285-299). Cambridge, MA: MIT Press.

BAMBER, D. (1975). The area above the ordinal dominance graph and the area below the receiver operating characteristic graph. Journal of Mathematical Psychology, 12, 387-415.

Blaisdell, A. P., \& CoOK, R. G. (2005). Two-item same-different concept learning in pigeons. Learning \& Behavior, 33, 67-77.

Brooks, D. I., \& Wasserman, E. A. (2008). Same/different discrimination learning with trial-unique stimuli. Psychonomic Bulletin \& Review, 15, 644-650.

Brooks, D. I., \& Wasserman, E. A. (2010). Contrasting object-based 
and texture-based accounts of same/different discrimination learning with trial-unique stimuli. Journal of Experimental Psychology: Animal Behavior Processes, 36, 158-163.

Castro, L., Kennedy, P. L., \& Wasserman, E. A. (2010). Conditional same-different discrimination by pigeons: Acquisition and generalization to novel and few-item displays. Journal of Experimental Psychology: Animal Behavior Processes, 36, 23-38.

Cook, R. G., \& BRooks, D. I. (2009). Generalized auditory samedifferent discrimination by pigeons. Journal of Experimental Psychology: Animal Behavior Processes, 35, 108-115.

Cook, R. G., Levison, D. G., Gillett, S. R., \& Blaisdell, A. P. (2005). Capacity and limits of associative memory in pigeons. Psychonomic Bulletin \& Review, 12, 350-358.

Delius, J. D. (1994). Comparative cognition of identity. In P. Bertelson, P. Eelen, \& G. d'Ydewalle (Eds.), International perspectives on psychological science: Vol. V. Leading themes (pp. 25-40). Hillsdale, NJ: Erlbaum.

Dittrich, L., Rose, J., Buschmann, J. U., Bourdonnais, M., \& GÜNTÜRKÜN, O. (2010). Peck tracking: A method for localizing critical features within complex pictures for pigeons. Animal Cognition, 13, 133-143.

Gibson, B. M., Wasserman, E. A., Frei, L., \& Miller, K. (2004). Recent advances in operant conditioning technology: A versatile and affordable computerized touchscreen system. Behavior Research Methods, Instruments, \& Computers, 36, 355-362.

Herrnstein, R. J., Loveland, D. H., \& Cable, C. (1976). Natural concepts in pigeons. Journal of Experimental Psychology: Animal Behavior Processes, 2, 285-302.

JENKINS, H. M., \& SAINSBURY, R. S. (1970). Discrimination learning with the distinctive feature on positive or negative trials. In D. I. Mostofsky (Ed.), Attention: Contemporary theory and analysis (pp. 239-273). New York: Appleton-Century-Crofts.

Katz, J. S., Wright, A. A., \& Bachevalier, J. (2002). Mechanisms of same/different abstract-concept learning by rhesus monkeys (Macaca mulatta). Journal of Experimental Psychology: Animal Behavior Processes, 28, 358-368

Katz, J. S., Wright, A. A., \& BodiLy, K. D. (2007). Issues in the comparative cognition of abstract-concept learning. Comparative Cognition \& Behavior Reviews, 2, 79-92.

LOCKE, J. (1975). An essay concerning human understanding. Oxford: Oxford University Press, Clarendon Press. (Original work published 1690)

McMurray, B., \& Aslin, R. N. (2005). Infants are sensitive to withincategory variation in speech perception. Cognition, 95, B15-B26.

Spivey, M. J., Grosjean, M., \& KNoblich, G. (2005). Continuous attraction toward phonological competitors. Proceedings of the National Academy of Sciences, 102, 10393-10398.

Tanenhaus, M. K., Spivey-Knowlton, M. J., Eberhard, K. M., \&
SEDIVy, J. C. (1995). Integration of visual and linguistic information in spoken language comprehension. Science, 268, 1632-1634.

Wasserman, E. A., \& ANDERSon, P. A. (1974). Differential autoshaping to common and distinctive elements of positive and negative discriminative stimuli. Journal of the Experimental Analysis of Behavior, 22, 491-496.

Wasserman, E. A., Fagot, J., \& Young, M. E. (2001). Same-different conceptualization by baboons (Papio papio): The role of entropy. Journal of Comparative Psychology, 115, 42-52.

Wasserman, E. A., Frank, A. J., \& Young, M. E. (2002). Stimulus control by same-versus-different relations among multiple visual stimuli. Journal of Experimental Psychology: Animal Behavior Processes, 28, 347-357.

Wasserman, E. A., Hugart, J. A., \& Kirkpatrick-Steger, K. (1995). Pigeons show same-different conceptualization after training with complex visual stimuli. Journal of Experimental Psychology: Animal Behavior Processes, 21, 248-252.

Wasserman, E. A., \& Young, M. E. (2010). Same-different discrimination: The keel and backbone of thought and reasoning. Journal of Experimental Psychology: Animal Behavior Processes, 36, 3-22.

Wasserman, E. A., Young, M. E., \& CoOK, R. G. (2004). Variability discrimination in humans and animals: Implications for adaptive action. American Psychologist, 59, 879-890.

Wasserman, E. A., Young, M. E., \& Peissig, J. J. (2002). Brief presentations are sufficient for pigeons to discriminate arrays of same and different stimuli. Journal of Experimental Psychology: Animal Behavior Processes, 78, 365-373.

Wright, A. A., \& KatZ, J. S. (2006). Mechanisms of same/different concept learning in primates and avians. Behavioral Processes, 72, 234-254.

Young, M. E., Ellefson, M. R., \& Wasserman, E. A. (2003). Toward a theory of variability discrimination: Finding differences. Behavioral Processes, 62, 145-155.

Young, M. E., \& Wasserman, E. A. (1997). Entropy detection by pigeons: Response to mixed visual displays after same-different discrimination training. Journal of Experimental Psychology: Animal Behavior Processes, 23, 157-170.

Young, M. E., \& Wasserman, E. A. (2001). Stimulus control in complex arrays. In R. G. Cook (Ed.), Avian visual cognition; available from www.pigeon.psy.tufts.edu/avc/young/.

Young, M. E., Wasserman, E. A., \& Garner, K. L. (1997). Effects of number of items on the pigeon's discrimination of same from different visual displays. Journal of Experimental Psychology: Animal Behavior Processes, 23, 491-501.

(Manuscript received July 20, 2009;

revision accepted for publication November 8, 2009.) 